\title{
Adapt-and-Distill: Developing Small, Fast and Effective Pretrained Language Models for Domains
}

\author{
Yunzhi Yao $^{\dagger}$, Shaohan Huang ${ }^{\ddagger}$, Wenhui Wang ${ }^{\ddagger}$, Li Dong ${ }^{\ddagger}$, Furu Wei ${ }^{\ddagger}$ \\ ${ }^{\dagger}$ Shandong University, Jinan, China \\ ${ }^{\ddagger}$ Microsoft Research, Beijing, China \\ yyz@mail.sdu. edu.cn \\ \{shaohanh, wenhui.wang, lidong1, fuwei\}@microsoft.com
}

\begin{abstract}
Large pretrained models have achieved great success in many natural language processing tasks. However, when they are applied in specific domains, these models suffer from domain shift and bring challenges in fine-tuning and online serving for latency and capacity constraints. In this paper, we present a general approach to developing small, fast and effective pretrained models for specific domains. This is achieved by adapting the offthe-shelf general pretrained models and performing task-agnostic knowledge distillation in target domains. Specifically, we propose domain-specific vocabulary expansion in the adaptation stage and employ corpus level occurrence probability to choose the size of incremental vocabulary automatically. Then we systematically explore different strategies to compress the large pretrained models for specific domains. We conduct our experiments in the biomedical and computer science domain. The experimental results demonstrate that our approach achieves better performance

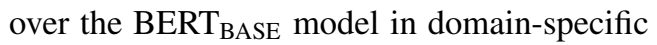
tasks while $3.3 \times$ smaller and $5.1 \times$ faster than BERT $_{\text {BASE. The code and pretrained models }}$ are available at https://aka.ms/adalm.
\end{abstract}

\section{Introduction}

Pre-trained language models, such as GPT (Radford et al., 2018), BERT (Devlin et al., 2019), RoBERTa (Liu et al., 2019) and UniLM (Dong et al., 2019) have achieved impressive success in many natural language processing tasks. These models usually have hundreds of millions of parameters. They are pre-trained on a large corpus of general domain and fine-tuned on target domain tasks. However, it is not optimal to deploy these models directly to edge devices in specific domains. First, heavy model size and high latency makes it difficult

\footnotetext{
${ }^{*}$ Contribution during internship at Microsoft Research.
}

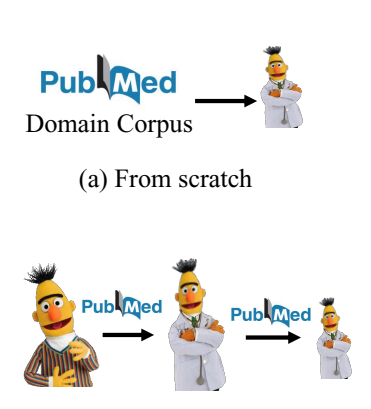

(c) Adapt-then-Distill

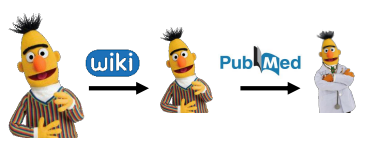

(b) Distill-then-Adapt

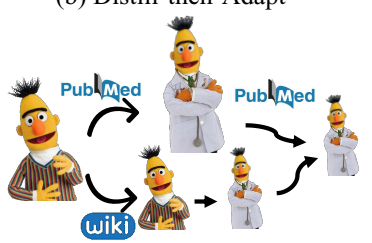

(d) Adapt-and-Distill
Figure 1: The four alternatives when distilling BERT into specific domains. All strategies are task-agnostic.

to deploy on resource-limited edge devices such as mobile phone. Second, directly fine-tuning a general pre-trained model on a domain-specific task may not be optimal when the target domain varies substantially from the general domain. Thirdly, many specialized domains contain their own specific terms, which are not included in pre-trained language model vocabulary.

In this paper, we introduce AdaLM, a framework that aims to develop small, fast and effective pretrained language models for specific domains. To address domain shift problem, recent studies (Lee et al., 2020; Gururangan et al., 2020) conduct continual pre-training to adapt a general domain pretrained model to specific domains. However, specific domains contain many common in-domain terms, which may be divided into bite-sized pieces (e.g., lymphoma is tokenized into [l, \#\#ym, \#\#ph, \#\#oma]). Gu et al.(2020) mentions that domainspecific vocabularies play a vital role in domain adaptation of pre-trained models. Specifically, we propose a domain-specific vocabulary expansion in the adaptation stage, which augments in-domain terms or subword units automatically given indomain text. Also, it is critical to decide the size of incremental vocabulary. Motivated by subword reg- 
ularization (Kudo, 2018), AdaLM introduces a corpus occurrence probability as a metric to optimize the size of incremental vocabulary automatically.

We systematically explore different strategies to compress general BERT models to specific domains (Figure 1): (a) From scratch: pre-training domain-specific small model from scratch with domain corpus; (b) Distill-then-Adapt: first distilling large model into small model, then adapting it into a specific domain; (c) Adapt-then-Distill: first adapting BERT into a specific domain, then distilling model into small size; (d) Adapt-and-Distill: adapting both the large and small models, then distilling with these two models initializing the teacher and student models respectively.

We conduct experiments in both biomedical and computer science domain and fine-tune the domainspecific small models on different downstream tasks. Experiments demonstrate that Adapt-andDistill achieves state-of-the-art results for domainspecific tasks. Specifically, the 6-layer model of 384 hidden dimensions outperforms the BERT $\mathrm{BASE}_{\mathrm{B}}$ model while $3.3 \times$ smaller and $5.1 \times$ faster than BERT $_{\text {BASE. }}$

\section{Related Work}

Domain adaptation of pre-trained model Most previous work on the domain-adaptation of pre-trained models targets large models. Lee et al. (2020) conduct continual pre-training to adapt the BERT model to the biomedical domain using the PubMed abstracts and the PMC full text. Gururangan et al. (2020) also employ continual pre-training to adapt pre-trained models into different domains including biomedical, computer science and news. However, many specialized domains contain their own specific words that are not included in pre-trained language model vocabulary. Gu et al.(2020) propose a biomedical pre-trained model PubMedBERT, where the vocabulary was created from scratch and the model is pre-trained from scratch. Furthermore, in many specialized domains, large enough corpora may not be available to support pre-training from scratch. Zhang et al. (2020) and Tai et al. (2020) extend the open-domain vocabulary with top frequent in-domain words to resolve this out-of-vocabulary issue. This approach ignores domain-specific sub-word units (e.g., blasto-, germin- in biomedical domain). These subword units help generalize domain knowledge and avoid unseen words

Task-agnostic knowledge distillation In recent years, tremendous progress has been made in model compression (Cheng et al., 2017). Knowledge distillation has proven to be a promising way to compress large models while maintaining accuracy (Sanh et al., 2019; Jiao et al., 2020; Sun et al., 2020; Wang et al., 2020). In this paper, we focus on task-agnostic knowledge distillation approaches, where a distilled small pre-trained model can be directly fine-tuned on downstream tasks. DistilBERT (Sanh et al., 2019) employs the soft label and embedding outputs to supervise the student. TinyBERT (Jiao et al., 2020) and MobileBERT (Sun et al., 2020) introduce self-attention distributions and hidden states to train the student model. MiniLM (Wang et al., 2020) avoids restrictions on the number of student layers and employs the self-attention distributions and value relation of the teacher's last transformer layer to supervise the student model. Because this method is more flexible, we implement MiniLM to compress large models in this work. No previous work systematically explores different strategies to achieve an effective and efficient smaller model in specific domains.

\section{Methods}

\subsection{Overview}

We systematically explore different strategies to achieve an effective and efficient small model in specific domains. We summarize them into four strategies: from scratch, distill-then-adapt, adaptthen-distill and adapt-and-distill.

Pretrain-from-scratch Domain-specific pretraining from scratch employs a random initialization of a pretrained model and pretrains a small model directly on domain-specific corpus. In this work, we conduct pretraining from scratch on different vocabularies including BERT original vocabulary, from scratch vocabulary, and expanded vocabulary.

Distill-then-adapt These approaches first distill the large general pretrained model which pretrained on Wikipedia and BookCorpus. Then it continues the pretraining process using a domain-specific corpus. In this work, we first distill the BERT model into a small model using task-agnostic knowledge distillation in MiniLM (Wang et al., 2020). Then 
we initialize the small model with it and conduct continual training with both the BERT original vocabulary and the expanded vocabulary.

Adapt-then-distill In this work, we select different large models as teacher models such as BERT and large models with different vocabularies. We first adapt these models into domain-specific models and then implement MiniLM to compress them to small models.

Adapt-and-distill In the previous part, when doing knowledge distill, we initialized the student model randomly. In order to get a better domainspecific small model, we try to explore the impact of the initialization of the student model. In this part, we adapt large and small models into specific domains separately, then use these two models to initialize the teacher and student model respectively.

\subsection{Domain Adaptation}

AdaLM contains a simple yet effective domain adaptation framework for a pretrained language model. As shown in Figure 2, it takes a general pretrained language model, original vocabulary and a domain specific corpus as input. Through vocabulary expansion and continual pretraining, AdaLM adapts general models into specific domains.

The core pipeline of domain adaptation consists of the three steps described below:

1. Given original vocabulary and a domainspecific corpus, the vocabulary expansion module aims to augment original vocabulary with domain-specific subword units or terms We augment domain-specific vocabulary from the target domain, while keeping the original BERT vocabulary unchanged. We describe them in more detail in Section 3.3.

2. Due to the size of the vocabulary having changed, we cannot initialize our model with BERT directly. As illustrated in Figure 3, we initialize the original embedding and Transformer encoder with weights from BERT (the green part in Figure 3). For incremental vocabulary, we first tokenize them into sub-words with the original vocabulary and then use an average pooling of their own sub-words embedding to initialize. As shown in Figure 3, the word 'lymphoma' is not included in BERT vocabulary. We tokenize it into three subwords (lym,\#\#pho, \#\#ma). The embedding
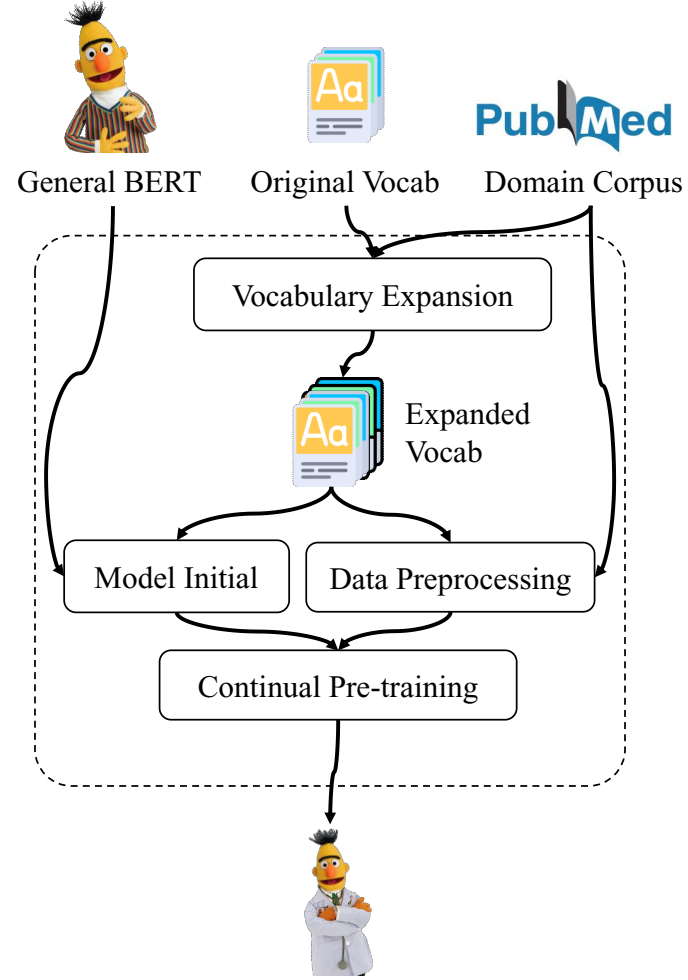

Figure 2: The pipeline of domain adaptation. Here we adapt the BERT model into the biomedical domain with PubMed dataset.

vector of 'lymphoma' is initialized by the average embedding vector of 'lym', '\#\#pho' and '\#\#ma'.

3. After model initialization and data preprocessing, we continually pretrain our model with domain-specific corpus using masked language model loss. Following BERT, we randomly replace $15 \%$ of tokens by a special token (e.g., [MASK]) and ask the language model to predict them in continual pretraining.

\subsection{Vocabulary Expansion}

Vocabulary expansion is the core module of AdaLM. It augments domain-specific terms or subword units to leverage domain knowledge. The size of the incremental vocabulary is a vital parameter for vocabulary expansion. Considering that unigram language modeling (Kudo, 2018) aligns more closely with morphology and avoids problems stemming from BPE's greedy construction procedure, as proposed in (Bostrom and Durrett, 2020), we followed Kudo (2018) and introduced a corpus occurrence probability as a metric to optimize the size of incremental vocabulary automati- 
Initial from Original BERT

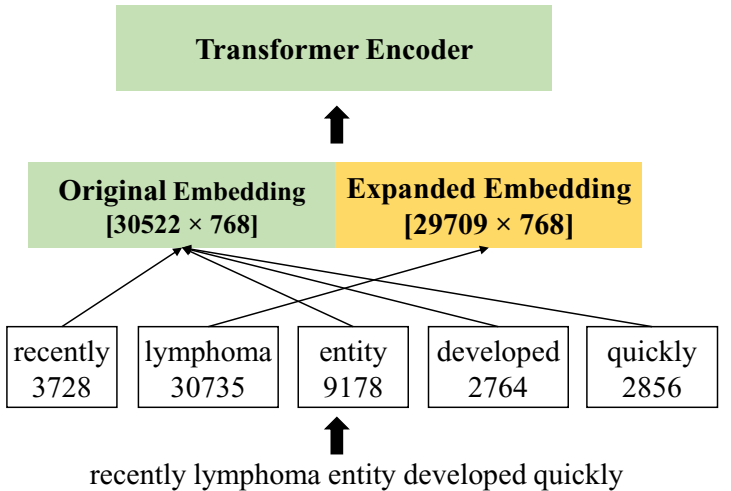

Figure 3: Concatenate original embedding with expanded embedding.

cally. We assume that each subword occurs independently and we assign to each subword in the corpus a probability equal to its frequency in the corpus.

$$
\forall i x_{i} \in \mathcal{V}, \sum_{x_{i} \in \mathcal{V}} p\left(x_{i}\right)=1,
$$

where $\mathcal{V}$ is a pre-determined vocabulary. The probability of a subword sequence $\mathbf{x}=\left(x_{1}, \ldots, x_{M}\right)$ can be computed by the product of the subword appearance probabilities $p\left(x_{i}\right)$. We convert it to logarithmic form:

$$
P(\mathbf{x})=\sum_{i=1}^{M} \log \left(p\left(x_{i}\right)\right),
$$

Given a domain-specific corpus $D$, the occurrence probability of corpus $D$ is formulated as:

$$
P(D)=\sum_{\mathbf{x}}^{|D|} \log (P(\mathbf{x})),
$$

where $\mathrm{x}$ represents tokenized sentence in corpus $D$.

We sample 550k sentences from the PubMed corpus and compute the occurrence probability $P(D)$ with different vocabulary sizes. The results are shown in Figure 4. We compare the occurrence probability with BERT and PubMedBERT vocabularies. We observe that $P(D)$ reveals a logarithmic trend with substantial increases at the beginning and little influence after vocabulary size of $70 \mathrm{k}$ in the biomedical domain. The PubMedBERT vocabulary performs similarly to the 40k size vocabulary. We present the occurrence probability of different vocabulary sizes in Appendix A.

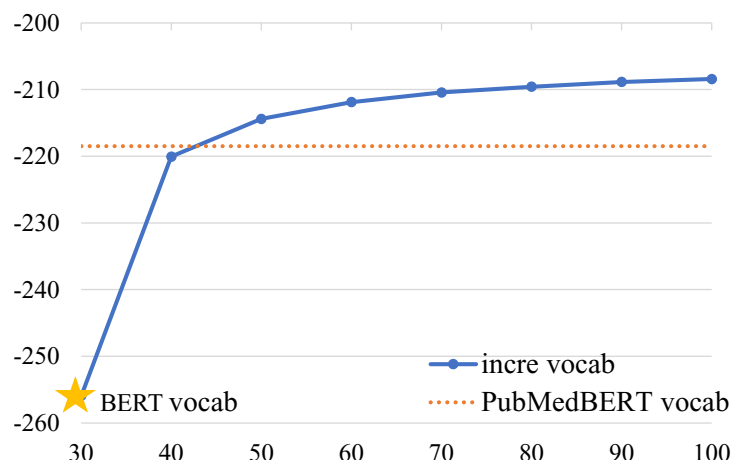

Figure 4: The $P(D)$ of different vocab sizes under biomedical domain. We use the BERT's vocabulary as the $30 \mathrm{k}$ vocabulary without vocabulary expanding. The PubMedBERT vocabulary is also $30 \mathrm{k}$.

We propose a simple method to decide the size of the incremental vocabulary. Assume the probability at the time step $i-1$ is $P_{i-1}(D)$ and at the time step $i$ is $P_{i}(D)$. If the rise $\frac{P_{i}(D)-P_{i-1}(D)}{P_{i-1}(D)}$ is lower than a threshold $\delta$, we regard the vocabulary size at the time step $i$ as the final size.

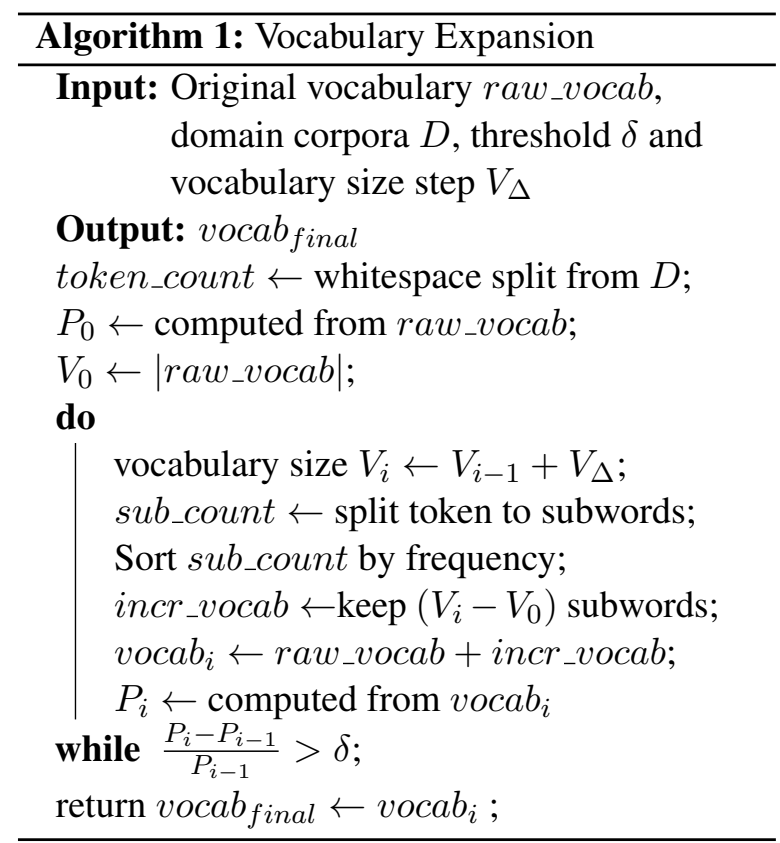

We expand the domain-specific vocabulary with the process shown in Algorithm 1. We implement our vocabulary expansion algorithm referring to SubwordTextBuilder in tensor2tensor ${ }^{1}$. In experiments, we set the threshold $\delta$ as $1 \%$ and vocabulary size step $V_{\Delta}$ as $10 \mathrm{k}$. Finally, we obtain the expanded vocabulary size of biomedical as $60 \mathrm{k}$ and computer science domain as 50k.

\footnotetext{
${ }^{1}$ https://github.com/tensorflow/tensor2tensor
} 


\section{Experiment Details}

We conduct our experiments in two domains: biomedical and computer science.

\subsection{Datasets}

Domain corpus: For the biomedical domain, we collect a 16GB corpus from PubMed ${ }^{2}$ abstracts to adapt our model. We use the latest collection and pre-process the corpora with the same process as PubMedBERT (we omit any abstracts with less than 128 words to reduce noise.).

For the computer science domain, we use the abstracts text from the arXiv ${ }^{3}$ Dataset. We select abstracts in computer science categories, collecting $300 \mathrm{M}$ entries for the corpus.

Fine-tuning tasks: For the biomedical domain, we choose three tasks: named entity recognition (NER), evidence-based medical information extraction (PICO), and relation extraction (RE). We perform entity-level F1 in NER task and wordlevel macro-F1 in the PICO task. The RE task uses the micro-F1 of positive classes evaluation. JNLPBA (Collier and Kim, 2004) NER dataset contains 6,892 disease mentions, which are mapped to 790 unique disease concepts with BIO tagging (Ramshaw and Marcus, 1995). EBM PICO (Nye et al., 2018) datasets annotates text spans with four tags: Participants, Intervention, Comparator and Outcome. ChemProt (Krallinger et al., 2017) dataset consists of five interactions between chemical and protein entities. We list the statistics of those tasks in Table 1.

We fine-tune two downstream tasks in the computer science domain. They are both classification tasks. The ACL-ARC (Jurgens et al., 2018) dataset mainly focuses on analyzing how scientific works frame their contributions through different types of citations. SCIERC (Luan et al., 2018) dataset includes annotations for scientific entities, their relations, and coreference clusters. The statistics are available in Table 2.

\subsection{Implementation}

We use the uncased version of BERT $\mathrm{BASE}_{\mathrm{BS}}$ (12 layers, 768 hidden size) as the large model and the MiniLM (6 layers, 384 hidden size) as the small model.

\footnotetext{
${ }^{2}$ https://pubmed.ncbi.nlm.nih.gov/

${ }^{3}$ https://www.kaggle.com/Cornell-University/arxiv

${ }^{4}$ https://microsoft.github.io/BLURB/
}

\begin{tabular}{lrrr}
\hline Dataset & Train & Dev & Test \\
\hline JNLPBA & 46,750 & 4551 & 8,662 \\
EBM PICO & 339,167 & 85321 & 16,364 \\
ChemProt & 18,035 & 11268 & 15,745 \\
\hline
\end{tabular}

Table 1: Biomedical dataset used in our experiment. All selected from BLURB ${ }^{4}$

\begin{tabular}{lcccc}
\hline Dataset & Train & Task & Test & Classes \\
\hline ACL-ARC & 1,688 & 114 & 139 & 6 \\
SCIERC & 3,219 & 455 & 974 & 7 \\
\hline
\end{tabular}

Table 2: Computer science dataset used in our experiment. We use the same train, development, and test splits as Gururangan et al. (2020)

To adapt the large model, we set the batch size at 8192 and the training step at 30,000. The peak learning rate was set to $6 \mathrm{e}-4$. To adapt the small model, we set the batch size as 256 and the training step as 200,000 . The learning rate is set to $1 \mathrm{e}-4$. The maximum length of the input sequence was 512 and the token masking probability was $15 \%$ for both the large model and the small model.

We implement MiniLM to compress large models and follow the setting of MiniLM, where the batch size was set to 256 and peak learning rate as $4 \mathrm{e}-4$. We set the training step as 200,000 .

For biomedical tasks, we follow the setting of PubMedBERT (Gu et al., 2020) to fine-tune these three tasks. For computer science tasks, we use the same setting as Gururangan et al. (2020). The concrete parameters are shown in Appendix B.

\section{Results}

The results of the tasks are shown in the Table 3 and 4 . We structure our evaluation by stepping through each of our three findings:

(1) Domain-specific vocabulary plays a significant role in domain-specific tasks and expanding vocabulary with the general vocabulary is better than just using domain-specific vocabulary.

We observe improved results via the expanded vocabulary with both the large and small models. For large model, AdaLM achieves the best results under each domain, where 77.74 on biomedical domain tasks, beating BioBERT and PubMedBERT and 77.76 on the computer science domain tasks.

For small models, in the biomedical domain, whether we train from scratch or distill-then-adapt with small models, incremental vocabulary mod- 


\begin{tabular}{|c|c|c|c|c|c|c|c|}
\hline Config & Type & Model & Teacher & JNLPBA & PICO & Chemprot & Average \\
\hline \multirow{4}{*}{$L=12 ; d=786$} & \multirow{4}{*}{ Large model } & BERT $^{\dagger}$ & - & 78.63 & 72.34 & 71.86 & 74.28 \\
\hline & & $\mathrm{BioBERT}^{\dagger}$ & - & 79.35 & 73.18 & 76.14 & 76.22 \\
\hline & & PubMedBERT $^{\dagger}$ & - & 80.06 & 73.38 & 77.24 & 76.89 \\
\hline & & AdaLM $^{\diamond}$ & - & 79.46 & 75.47 & 78.41 & 77.74 \\
\hline \multirow{11}{*}{$L=6 ; d=384$} & Small model & MiniLM & - & 77.44 & 71.69 & 68.08 & 72.40 \\
\hline & \multirow{3}{*}{ From scratch } & BERT vocab (a) & - & 77.89 & 72.97 & 70.21 & 73.69 \\
\hline & & PubMed vocab (b) & - & 77.82 & 73.82 & 70.32 & 73.99 \\
\hline & & AdaLM vocab (c) & - & 77.80 & 73.39 & 70.86 & 74.02 \\
\hline & \multirow{3}{*}{ Distill-then-Adapt } & BERT vocab (d) & - & 78.63 & 74.00 & 72.28 & 74.97 \\
\hline & & PubMed vocab (e) & - & 78.36 & 73.91 & 71.33 & $\overline{74.53}$ \\
\hline & & AdaLM vocab (f) & - & 78.77 & 74.23 & 72.29 & $\underline{75.10}$ \\
\hline & \multirow{3}{*}{ Adapt-then-Distill } & Random initial (g) & BERT & 77.98 & 72.38 & 68.86 & 73.07 \\
\hline & & Random initial (h) & PubMedBERT & 78.78 & 74.20 & 70.89 & $\underline{74.62}$ \\
\hline & & Random initial (i) & AdaLM $^{\diamond}$ & 78.98 & 74.78 & 71.51 & $\overline{75.09}$ \\
\hline & Adapt-and-Distill & Model (f) initial (j) & AdaLM ${ }^{\diamond}$ & 79.04 & 74.91 & 72.06 & $\underline{75.34}$ \\
\hline
\end{tabular}

Table 3: Comparison between different strategies on biomedical tasks. The AdaLM ${ }^{\diamond}$ means we just adapt the large model without distillation. Scores of the methods marked with ${ }^{\dagger}$ are taken from (Gu et al., 2020). Underlined data marks the small models whose performances surpass the BERT model's performance. $L$ and $d$ indicate the number of layers and the hidden dimension of the model.

els always perform better than the general vocabulary or just the domain-specific vocabulary. (When distill-then-adapt with the PubMed vocabulary, we initialize the word embedding in the same way as mentioned in Section 3.2). In addition, with distillthen-adapt, the model (f) (75.10) can surpass the BERT model (74.28).

In the computer science domain, distill-thenadapt models with incremental vocabulary also show great performance. Model (d) achieves a comparable result of 72.91 as BERT and outperforms BERT in the ACL-ARC datasets with 65.93 $(+1.01 \mathrm{~F} 1)$. We also observe that when training from scratch, the results of Model (b) with incremental vocabulary are lower (1.45 lower) than that of model (a). This may be because after vocabulary expansion, a from-scratch model needs to be pretrained with more unlabeled data.

(2) Continual pretraining on domain-specific texts from general language models is better than pretraining from scratch.

$\mathrm{Gu}$ et al. (2020) finds that for domains with abundant unlabeled texts, pretraining language models from scratch outperforms continual pretraining of general-domain language models. However, in our experiments, we find that general-domains model can help our model to learn the target domain better. In the biomedical domain, we use MiniLM model to initialize the model (d), (e) and (f) in distill-thenadapt setting. No matter which vocabulary is used, continual pretraining on domain-specific texts from general language models is better than pretraining from scratch. For AdaLM vocabulary, the model (f) gets 75.10, outperforming the model (c) trained from scratch with the same vocabulary by 1.08 . On the other hand, for domains that do not have enormous unlabeled texts such as the computer science domain in our experiments, continual pretraining also showed better results. With continual pretraining, model (d) achieves higher results exceeding both model (b) (+5.66 F1) and model (c) $(+0.47$ F1).

(3) Adapt-and-Distill is the best strategy to develop a task-agnostic domain-specific small pretrained model.

In the Adapt-then-Distill part, our findings supports evidence from previous observations (Wang et al., 2020) that a better teacher model leads to a better student model. Using AdaLM which performs best among large models as the teacher model can yield good results: 75.09 in the biomedical domain and 71.62 in the computer science, better than other domain-specific large models. Furthermore, we find that a better student model for initialization can also help to get a better small model. In the Adapt-and-Distill part, we adapt large and small models into specific domains separately and then compress the adapted large model as the teacher with the adapted small model as initialization. In the biomedical domain, the model (j), initialized from model (i), achieves the best result of 75.34 among the small models. It also 


\begin{tabular}{|c|c|c|c|c|c|c|}
\hline Config & Type & Model & Teacher & ACL-ARC & SCIERC & Average \\
\hline \multirow{2}{*}{$L=12 ; d=786$} & \multirow{2}{*}{ Large model } & BERT & - & 64.92 & 81.14 & 73.03 \\
\hline & & AdaLM $^{\diamond}$ & - & 73.61 & 81.91 & 77.76 \\
\hline \multirow{8}{*}{$L=6 ; d=384$} & Small model & MiniLM & - & 61.5 & 72.88 & 67.19 \\
\hline & \multirow{2}{*}{ From scratch } & BERT vocab (a) & - & 62.48 & 74.93 & 68.70 \\
\hline & & AdaLM vocab (b) & - & 59.57 & 74.93 & 67.25 \\
\hline & \multirow{2}{*}{ Distill-then-Adapt } & BERT vocab (c) & - & 65.75 & 79.13 & 72.44 \\
\hline & & AdaLM vocab (d) & - & 65.93 & 79.88 & 72.91 \\
\hline & \multirow{2}{*}{ Adapt-then-Distill } & Random initial (e) & BERT & 63.12 & 77.89 & 70.50 \\
\hline & & Random initial (f) & AdaLM $^{\diamond}$ & 66.21 & 77.04 & 71.62 \\
\hline & Adapt-and-Distill & Model (d) initial (g) & AdaLM $^{\diamond}$ & 68.74 & 78.88 & $\underline{73.81}$ \\
\hline
\end{tabular}

Table 4: Comparison between different strategies on computer science tasks. The AdaLM ${ }^{\diamond}$ is the adapted large model without compressing. We report averages across five random seeds. Data marked with underlines are the results of small models which outperform the BERT model's. $L$ and $d$ indicate the number of layers and the hidden dimension of the model.

outperforms the BERT model (+1.06 F1). In the computer science domain, model (g), initialized by model (d), is the only small model that outperforms BERT (+0.78 F1).

\section{Analysis}

\subsection{Inference Speed}

We compare AdaLM's parameters' size and inference speed with the BERT model in the biomedical domain in Table 5.

\begin{tabular}{llcc}
\hline Type & Model & \#Params & Speedup \\
\hline \multirow{2}{*}{ Large } & BERT & $109 \mathrm{M}$ & $\times 1.0$ \\
& PubMedBERT & $109 \mathrm{M}$ & $\times 1.0$ \\
& AdaLM vocab & $132 \mathrm{M}$ & $\times 1.07$ \\
\hline \multirow{2}{*}{ Small } & BERT vocab & $22 \mathrm{M}$ & $\times 5.0$ \\
& AdaLM & $34 \mathrm{M}$ & $\times 5.1$ \\
\hline
\end{tabular}

Table 5: Comparison of model's parameter size and the inference speed. The inference speedup is computed by the classification task ChemProt and evaluated on a single NVIDIA P100 GPU.

First we can find that the vocabulary expansion yields marginal improvements on the model's inference speed. We added about $20 \mathrm{M}$ parameters in the embedding weights in the large model using AdaLM vocabulary, but its inference speed is slightly faster than BERT and PubMedBERT. Since most domain-specific terms are shattered into fragmented subwords, the length of the token sequence we get by using the incremental vocabulary is shorter than the length of the sequence got by the original vocabulary, which reduces the computation load. We list the change of the sequence length of the downstream tasks in Appendix C. Meanwhile, in the embedding layers, the model just needs to map the sub-words' id to their dense representations, which is little affected by the parameters' size. The small model shows the same trend.

In addition, the small model AdaLM shows great potential. Compared with the 12-layer model of 768 hidden dimensions, the 6-layer model of 384 hidden dimensions is $3.3 \mathrm{x}$ smaller and 5.1 $\mathrm{x}$ faster in the model efficiency, while performing similarly to or even better than BERT $\mathrm{BASE}$.

\subsection{Impact of Training Time}

Pre-training often demands lots of time. In this section, we examine the adapted model's performance as a function of training time. Here we use the biomedical domain since its unlabelled texts are abundant and compare the large domain-specific adapted model with BioBERT. For every $24 \mathrm{hrs}$ of continual pre-training, we fine-tuned the adapted model on the downstream tasks. For comparison, we convert the training time of BioBERT to the time it may take with the same computing resource of this work (16 V100 GPUs).

We list the results in Table 6, we denote the large adapted model as AdaLM in the table. AdaLM at 0 hrs means that we fine-tune the initialized model directly without any continual pre-training. We find that BERT is slightly better than 0hr AdaLM and after 24 hrs, AdaLM outperforms BioBERT, which demonstrates that domain-specific vocabulary is very critical for domain adaption of pre-trained model. Our experiments demonstrate promising re- 


\begin{tabular}{lcc}
\hline Model & Training Time & Average \\
\hline & $0 \mathrm{hrs}$ & 74.25 \\
AdaLM & $24 \mathrm{hrs}$ & 76.80 \\
& $48 \mathrm{hrs}$ & 77.36 \\
& $72 \mathrm{hrs}$ & 77.74 \\
\hline BERT & $0 \mathrm{hrs}$ & 74.28 \\
\hline BioBERT & $120 \mathrm{hrs}$ & 76.22 \\
\hline
\end{tabular}

Table 6: Results with different pre-training time. In the table, AdaLM is the adapted large model without compressing.

sults in the biomedical domain. Under constrained computation, AdaLM achieves better performance compared to BioBERT. More details can be found in Appendix D

\subsection{Impact of Vocabulary Size}

To understand the impact of the vocabulary size, we conduct some experiments with different vocabulary sizes in the biomedical domain. We select the biomedical large AdaLM model and to reduce the computation load, we set the batch size as 256 and step as $250 \mathrm{~K}$ in our ablation studies. We show performance of the model with different sizes in Table 7.

\begin{tabular}{lccccc}
\hline & $40 \mathrm{k}$ & $50 \mathrm{k}$ & $60 \mathrm{k}$ & $70 \mathrm{k}$ & $80 \mathrm{k}$ \\
\hline JNLPBA & 78.84 & $\mathbf{7 9 . 0 2}$ & 78.91 & 78.94 & 79.01 \\
\hline PICO & $\mathbf{7 5 . 0 9}$ & 74.81 & 74.99 & 74.58 & 75.00 \\
\hline ChemProt & 76.10 & 76.80 & $\mathbf{7 7 . 2 1}$ & 76.40 & 76.85 \\
\hline Average & 76.67 & 76.87 & $\mathbf{7 7 . 0 3}$ & 76.64 & 76.95 \\
\hline
\end{tabular}

Table 7: The performance of different vocabulary sizes

We observe that the model of $60 \mathrm{k}$ achieves the best results in our ablation studies. The result is a bit surprising. Despite having a larger vocabulary, the $70 \mathrm{k}$ and $80 \mathrm{k}$ model does not show a stronger performance. A possible explanation for these results may be that a larger vocabulary set may contain some more complicated but less frequent words, which cannot be learnt well through continual pre-training. For example, the word ferrocytochrome exists in $70 \mathrm{k}$ and $80 \mathrm{k}$ vocabularies but is split into ('ferrocy', '\#\#tochrom', '\#\#e') in the $60 \mathrm{k}$ vocabulary. In our sampled data (about 550k sentences), 'ferrocytochrome' appears less than 100 times, while the subword '\#\#tochrom' appears more than 10k times and 'ferrocy' appears more than 200 times. The representation of those rare words cannot be learnt well due to the sparsity problem.

\subsection{Vocabulary Visualization}

The main motivation for using an the expanded vocabulary set is to leverage domain knowledge better. Compared to PubMedBERT which just uses the domain-specific vocabulary and initializes the model randomly, the keep of the general vocabulary and the general language model's weights may help us make good use of the existing knowledge and word embedding.

To assess the importance of the expanded vocabulary, we compute the L2-distance of the embedding weights before and after pre-training in our AdaLM model in the biomedical domain in Figure 5.

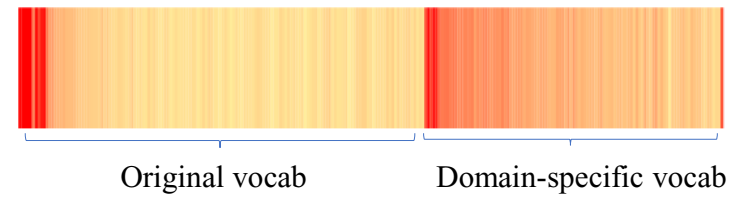

Figure 5: The L2-distance of the embedding layer. The deeper the color, the farther the distance.

We observe that the domain-specific vocabulary part changes a lot during the pre-training time, which indicates that our model learns much information about these domain-specific terms. We also observe that there is little change in many original sub-words' embedding weights, which indicates that many general vocabularies can be used directly in continual training.

\section{Conclusion}

In this paper, we investigate several variations to compress general BERT models to specific domains. Our experiments reveal that the best strategy to obtain a task-agnostic domain-specific pretrained model is to adapt large and small models into specific domains separately and then compress the adapted large model with the adapted small model as initialization. We show that the adapted 6layer model of 384 hidden dimensions outperforms the BERT $_{\text {BASE }}$ model while $3.3 \times$ smaller and $5.0 \times$ faster than $\mathrm{BERT}_{\mathrm{BASE}}$. Our findings suggest that domain-specific vocabulary and general-domain language model play vital roles in domain adaptation of a pretrained model. In the future, we will investigate more directions in domain adaptation, such as data selection and efficient adaptation. 


\section{References}

Kaj Bostrom and Greg Durrett. 2020. Byte pair encoding is suboptimal for language model pretraining. In Findings of the Association for Computational Linguistics: EMNLP 2020, pages 4617-4624, Online. Association for Computational Linguistics.

Yu Cheng, Duo Wang, Pan Zhou, and Tao Zhang. 2017. A survey of model compression and acceleration for deep neural networks. arXiv preprint arXiv:1710.09282.

Nigel Collier and Jin-Dong Kim. 2004. Introduction to the bio-entity recognition task at JNLPBA In Proceedings of the International Joint Workshop on Natural Language Processing in Biomedicine and its Applications (NLPBA/BioNLP), pages 73-78, Geneva, Switzerland. COLING.

Jacob Devlin, Ming-Wei Chang, Kenton Lee, and Kristina Toutanova. 2019. BERT: Pre-training of deep bidirectional transformers for language understanding. In Proceedings of the 2019 Conference of the North American Chapter of the Association for Computational Linguistics: Human Language Technologies, Volume 1 (Long and Short Papers), pages 4171-4186, Minneapolis, Minnesota. Association for Computational Linguistics.

Li Dong, Nan Yang, Wenhui Wang, Furu Wei, Xiaodong Liu, Yu Wang, Jianfeng Gao, Ming Zhou, and Hsiao-Wuen Hon. 2019. Unified language model pre-training for natural language understanding and generation. In Advances in Neural Information Processing Systems 32: Annual Conference on Neural Information Processing Systems 2019, NeurIPS 2019, December 8-14, 2019, Vancouver, $B C$, Canada, pages 13042-13054.

Yu Gu, Robert Tinn, Hao Cheng, Michael Lucas, Naoto Usuyama, Xiaodong Liu, Tristan Naumann, Jianfeng Gao, and Hoifung Poon. 2020. Domainspecific language model pretraining for biomedical natural language processing. arXiv preprint arXiv:2007.15779.

Suchin Gururangan, Ana Marasović, Swabha Swayamdipta, Kyle Lo, Iz Beltagy, Doug Downey, and Noah A. Smith. 2020. Don't stop pretraining: Adapt language models to domains and tasks. In Proceedings of the 58th Annual Meeting of the Association for Computational Linguistics, pages 8342-8360, Online. Association for Computational Linguistics.

Xiaoqi Jiao, Yichun Yin, Lifeng Shang, Xin Jiang, Xiao Chen, Linlin Li, Fang Wang, and Qun Liu. 2020. TinyBERT: Distilling BERT for natural language understanding. In Findings of the Association for Computational Linguistics: EMNLP 2020, pages 4163-4174, Online. Association for Computational Linguistics.
David Jurgens, Srijan Kumar, Raine Hoover, Dan McFarland, and Dan Jurafsky. 2018. Measuring the evolution of a scientific field through citation frames. Transactions of the Association for Computational Linguistics, 6:391-406.

Martin Krallinger, Obdulia Rabal, Saber A Akhondi, Martın Pérez Pérez, Jesús Santamaría, GP Rodríguez, et al. 2017. Overview of the biocreative vi chemical-protein interaction track. In Proceedings of the sixth BioCreative challenge evaluation workshop, volume 1, pages 141-146.

Taku Kudo. 2018. Subword regularization: Improving neural network translation models with multiple subword candidates. In Proceedings of the 56th Annual Meeting of the Association for Computational Linguistics (Volume 1: Long Papers), pages 66-75, Melbourne, Australia. Association for Computational Linguistics.

Jinhyuk Lee, Wonjin Yoon, Sungdong Kim, Donghyeon Kim, Sunkyu Kim, Chan Ho So, and Jaewoo Kang. 2020. Biobert: a pre-trained biomedical language representation model for biomedical text mining. Bioinformatics, 36(4):1234-1240.

Yinhan Liu, Myle Ott, Naman Goyal, Jingfei Du, Mandar Joshi, Danqi Chen, Omer Levy, Mike Lewis, Luke Zettlemoyer, and Veselin Stoyanov. 2019. Roberta: A robustly optimized bert pretraining approach. arXiv preprint arXiv:1907.11692.

Yi Luan, Luheng He, Mari Ostendorf, and Hannaneh Hajishirzi. 2018. Multi-task identification of entities, relations, and coreference for scientific knowledge graph construction. In Proceedings of the 2018 Conference on Empirical Methods in Natural Language Processing, pages 3219-3232, Brussels, Belgium. Association for Computational Linguistics.

Benjamin Nye, Junyi Jessy Li, Roma Patel, Yinfei Yang, Iain Marshall, Ani Nenkova, and Byron Wallace. 2018. A corpus with multi-level annotations of patients, interventions and outcomes to support language processing for medical literature. In Proceedings of the 56th Annual Meeting of the Association for Computational Linguistics (Volume 1: Long Papers), pages 197-207, Melbourne, Australia. Association for Computational Linguistics.

Alec Radford, Karthik Narasimhan, Tim Salimans, and Ilya Sutskever. 2018. Improving language understanding by generative pre-training.

Lance Ramshaw and Mitch Marcus. 1995. Text chunking using transformation-based learning. In Third Workshop on Very Large Corpora.

Victor Sanh, Lysandre Debut, Julien Chaumond, and Thomas Wolf. 2019. Distilbert, a distilled version of bert: smaller, faster, cheaper and lighter. arXiv preprint arXiv:1910.01108. 
Zhiqing Sun, Hongkun Yu, Xiaodan Song, Renjie Liu, Yiming Yang, and Denny Zhou. 2020. MobileBERT: a compact task-agnostic BERT for resource-limited devices. In Proceedings of the 58th Annual Meeting of the Association for Computational Linguistics, pages 2158-2170, Online. Association for Computational Linguistics.

Wen Tai, HT Kung, Xin Luna Dong, Marcus Comiter, and Chang-Fu Kuo. 2020. exbert: Extending pretrained models with domain-specific vocabulary under constrained training resources. In Proceedings of the 2020 Conference on Empirical Methods in Natural Language Processing: Findings, pages 1433-1439.

Wenhui Wang, Furu Wei, Li Dong, Hangbo Bao, Nan Yang, and Ming Zhou. 2020. Minilm: Deep selfattention distillation for task-agnostic compression of pre-trained transformers. In Advances in Neural Information Processing Systems 33: Annual Conference on Neural Information Processing Systems 2020, NeurIPS 2020, December 6-12, 2020, virtual.

Rong Zhang, Revanth Gangi Reddy, Md Arafat Sultan, Vittorio Castelli, Anthony Ferritto, Radu Florian, Efsun Sarioglu Kayi, Salim Roukos, Avi Sil, and Todd Ward. 2020. Multi-stage pre-training for low-resource domain adaptation. In Proceedings of the 2020 Conference on Empirical Methods in Natural Language Processing (EMNLP), pages 54615468, Online. Association for Computational Linguistics. 


\section{A Occurrence probability of different vocabulary sizes}

\begin{tabular}{lc}
\hline Vocabulary & P(D) \\
\hline BERT & -255.92 \\
PubMed & -218.49 \\
\hline 40k vocab & -220.06 \\
50k vocab & -214.40 \\
60k vocab & -211.88 \\
70k vocab & -210.44 \\
80k vocab & -209.57 \\
90k vocab & -208.86 \\
100k vocab & -208.42 \\
\hline
\end{tabular}

Table 8: The $P(D)$ of different vocabulary under biomedical domain.

\begin{tabular}{lc}
\hline Vocabulary & P(D) \\
\hline BERT & -211.14 \\
\hline 40k vocab & -194.08 \\
50k vocab & -192.56 \\
60k vocab & -191.87 \\
70k vocab & -191.45 \\
80k vocab & -191.09 \\
90k vocab & -190.76 \\
100k vocab & -190.53 \\
\hline
\end{tabular}

Table 9: The $P(D)$ of different vocabulary under computer science domain.

\section{B Fine-tuning hyperparameters for downstream tasks}

\begin{tabular}{llcc}
\hline Hyperameter & \multicolumn{3}{c}{ Assignment } \\
\hline & NER & PICO & RE \\
\hline Batch size & 32 & \multicolumn{3}{c}{16,32} & 32 \\
Learning rate & \multicolumn{3}{c}{$1 \mathrm{e}-5,3 \mathrm{e}-5,5 \mathrm{e}-5$} \\
Epoch & $\{30-40\}$ & $\{10,15\}$ & $\{40-50\}$ \\
Dropout & \multicolumn{3}{c}{0.1} \\
\hline
\end{tabular}

Table 10: Hyparameters we used to finetune on biomedical tasks.

\section{Sequence Length}

After the vocabulary expansion, the length of the token sequence may get shorter. We compute the average sentence length of the downstream tasks. We list the results in Table 12

\begin{tabular}{lc}
\hline Hyperameter & Assignment \\
\hline & ACL-ARC $\quad$ SCIERC \\
\hline Batch size & 16 \\
Learning rate & $2 \mathrm{e}-5$ \\
Epoch & 20 \\
Dropout & 0.1 \\
\hline
\end{tabular}

Table 11: Hyparameters we used to finetune on computer science tasks.

\begin{tabular}{lcc}
\hline Dataset & Original Vocab & Incr. Vocab \\
\hline ChemProt & 66 & 53 \\
EBM PICO & 36 & 31 \\
JNLPBA & 41 & 32 \\
\hline ACL-ARC & 53 & 50 \\
SCIERC & 45 & 42 \\
\hline
\end{tabular}

Table 12: The sequence length tokenized by the original vocabulary and expanded vocabulary.

\section{Results of different training time}

We list the biomedical tasks' results of each pretraining time in the following table.

\begin{tabular}{lcccc}
\hline & $\mathbf{0 h}$ & $\mathbf{2 4 h}$ & $\mathbf{4 8 h}$ & $\mathbf{7 2 h}$ \\
\hline JNLPBA & 77.56 & 79.14 & 79.11 & 79.46 \\
\hline PICO & 73.29 & 74.22 & 75.28 & 75.36 \\
\hline ChemProt & 71.91 & 77.06 & 77.69 & 78.42 \\
\hline Average & 74.25 & 76.80 & 77.36 & 77.74 \\
\hline
\end{tabular}

Table 13: The performance of models with different pretraining time 